\title{
A Honeycomb Shape Localization Algorithm Based on Levy-PSO
}

\author{
Dongyao Zou' ${ }^{1}$, Biwei Liu ${ }^{1}$ and Chen $\mathrm{Li}^{1}$
}

${ }^{1}$ College of Computer and Communication Engineering, Zhengzhou University of Light Industry, Zhengzhou 450001, China

1574990296@qq.com

Keywords. WSNs,particle swarm optimization(PSO), honeycomb,the least squares fitting,Levy flight mechanism

\begin{abstract}
The positioning technology is of vital importance in Wireless Sensor Networks (WSNs), and its accuracy based on particle swarm optimization (PSO) is low. Therefore, this paper considers the problem of low positioning accuracy and slow speed of convergence by proposing a honeycomb shape localization algorithm based on Levy-PSO. First, the communication distance and ranging error analysis shows that there is an optimal length can improve localization accuracy. Then, the localization area is divided into several honeycomb sub-regions with the optimal length. The unknown node communicates with at least six beacon nodes to ensure the reliability of the results. And these honeycomb sub-regions develop their environmental parameters which are calculated from the least squares fitting method. Last, we develop the PSO algorithm with Levy flight mechanism. The simulation results are show the way this algorithm could improve the positioning accuracy in order to have better stability,
\end{abstract}

\section{Introduction}

With low cost and power consumption advantages, the distributed and self-organization, wireless sensor network has rapid development. Due to Wireless sensor network is an event monitoring network, self-localization of senor nodes is a highly desirable feature, where the collected data are insignificant without getting the location of these data [1]-[3]. Because the positional information are not only obtain where data are coming from, but track a moving target and predict it direction. It's urgent to study how we can more accurately grasp the location of target.

In the case of the node estimation mechanism, positioning algorithm has two categories: one is range-based, the other one is range-free [4], which are divided by whether the positioning process needs to measure the actual distance or angle between the nodes. In the range-based algorithm, the methods of measuring the distance or orientation between nodes are TOA (Time of Arrival), RSSI (Received Signal Strength Indication) and AOA (Angle of Arrival) [5-6]. In recent years, the researchers used the genetic algorithm, the least square method, the particle swarm optimization algorithm for the research of node localization technology in wireless sensor network [7-10]. Due to particle swarm optimization algorithm has problems of slow convergence speed, fall into local minimum point easily and premature convergence, which makes difficult for positioning performance promotion.

\section{The Improved Algorithm Designs}

From analyzing the triangle centroid algorithm, this algorithm concludes three stages: the analysis of communication distance and ranging error; the division of positioning area with optimal communication radius; the Levy-PSO localization.

2.1 The Division of Positioning Area. The positioning area is divided into honeycomb regions, which beacon nodes are distributed on the vertex of sub-region. The layout shows in Figure 1 . The coordinates of beacon nodes are set as (1) and (2), in which $X$ is the value of horizontal axis and $Y$ is the value of vertical axis. 


$$
X=\left[\begin{array}{cccccc}
\frac{1}{2} & \frac{3}{2} & \cdots & \frac{1+n}{2} & \cdots & \frac{1+N}{2} \\
0 & 2 & \cdots & 2 n & \cdots & 2 N \\
1 & 3 & \cdots & \frac{1+n}{2} & \cdots & \frac{1+N}{2} \\
2 & 2 & \cdots & 0 & \cdots & 2 N \\
0 & 2 & \cdots & 2 n & \cdots & 2 N \\
\vdots & & & & & \\
0 & 2 & \cdots & 2 n & \cdots & 2 N
\end{array}\right]
$$$$
\text { (1) } Y=\left[\begin{array}{cccccc}
\frac{\sqrt{3}}{2} & \frac{\sqrt{3}}{2} & \cdots & \frac{\sqrt{3}}{2} & \cdots & \frac{\sqrt{3}}{2} \\
\sqrt{3} & \sqrt{3} & \cdots & \sqrt{3} & \cdots & \sqrt{3} \\
\vdots & & & & & \\
\frac{\sqrt{3} n}{2} & \frac{\sqrt{3} n}{2} & \cdots & \frac{\sqrt{3} n}{2} & \cdots & \frac{\sqrt{3} n}{2} \\
\vdots & & & & & \\
\frac{\sqrt{3} N}{2} & \frac{\sqrt{3} N}{2} & \cdots & \frac{\sqrt{3} N}{2} & \cdots & \frac{\sqrt{3} N}{2}
\end{array}\right]
$$

We already know the communication distance has relation to ranging error, so the sub-regional length of honeycomb use the optimal value $-5 \mathrm{~m}$. As a result, the unknown node communicates with at least six beacon nodes.

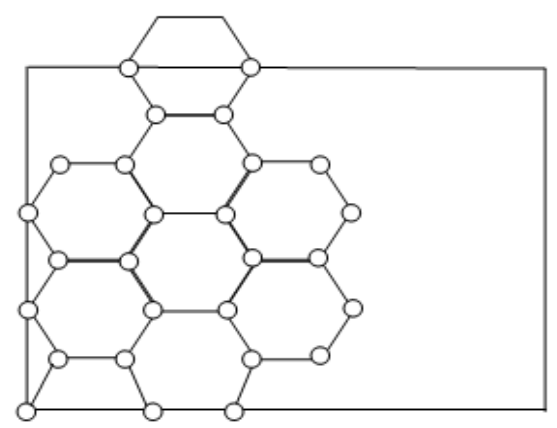

Figure 1. The honeycomb shape layout of beacon nodes

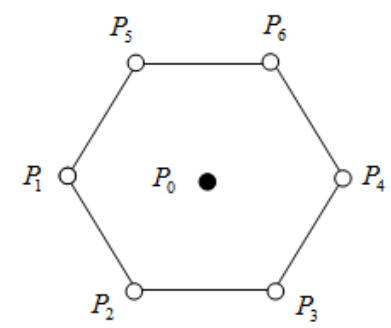

Figure 2. The nodes distribution of parameters fitting

As is shown in Figure 2, the environmental parameters of sub-region develop the least squares fitting. Where $P_{0}$ is the unknown node, placed in the centroid position of honeycomb sub-region. The experimental data is the mean of RSSI value getting from beacon nodes $P_{1} \sim P_{6}$. Establish a table that is the relationship between beacon nodes and sub-regional environmental parameters. The steps of sub-regional fitting as follows:

(1) The number of sampling points sets $m$; sampling points are $\left(x_{i}, y_{i}\right)(i=1,2, \cdots, m)\left(y_{i}\right.$ is the RSSI value when $x$ is $x_{i}$ );

(2) The fitting function $p(x)$ contains a group of continuous functions $g_{0}(x), g_{1}(x), \cdots g_{s}(x)$ which are linearly independent: $p(x)=a_{0} g_{0}(x)+a_{1} g_{1}(x)+\cdots+a_{s} g_{s}(x)$. Where $a_{1}, a_{2}, \cdots a_{s}$ are coefficient, plug into $\left[\begin{array}{llll}x_{1} & x_{2} & \cdots & x_{m}\end{array}\right]^{-1}$, we can get

$$
P(x)=\left[\begin{array}{c}
a_{0} g_{0}\left(x_{1}\right)+a_{1} g_{1}\left(x_{1}\right)+a_{2} g_{2}\left(x_{1}\right)^{2}+\cdots+a_{s} g_{s}\left(x_{1}\right)^{s} \\
a_{0} g_{0}\left(x_{2}\right)+a_{1} g_{1}\left(x_{2}\right)+a_{2} g_{2}\left(x_{2}\right)^{2}+\cdots+a_{s} g_{s}\left(x_{2}\right)^{s} \\
\vdots \\
a_{0} g_{0}\left(x_{m}\right)+a_{1} g_{1}\left(x_{m}\right)+a_{2} g_{2}\left(x_{m}\right)^{2}+\cdots+a_{s} g_{s}\left(x_{m}\right)^{s}
\end{array}\right]
$$

(3) Establish a bias squares function: $J\left(a_{1}, a_{2}, \cdots a_{s}\right)$;

$$
J=\sum_{i=0}^{m}\left[P\left(x_{i}\right)-y_{i}\right]^{2}=S U M\left[\begin{array}{c}
a_{0} g_{0}\left(x_{1}\right)+a_{1} g_{1}\left(x_{1}\right)+a_{2} g_{2}\left(x_{1}\right)+\cdots+a_{s} g_{s}\left(x_{1}\right)-y_{1} \\
a_{0} g_{0}\left(x_{2}\right)+a_{1} g_{1}\left(x_{2}\right)+a_{2} g_{2}\left(x_{2}\right)+\cdots+a_{s} g_{s}\left(x_{2}\right)-y_{2} \\
\vdots \\
a_{0} g_{0}\left(x_{m}\right)+a_{1} g_{1}\left(x_{m}\right)+a_{2} g_{2}\left(x_{m}\right)+\cdots+a_{s} g_{s}\left(x_{m}\right)-y_{m}
\end{array}\right]^{2}
$$

(4) There is a system of linear equations about $a_{1}, a_{2}, \cdots a_{s}$.To solve function $J$ of the minimum value is the equal of $a_{1}, a_{2}, \cdots a_{s}$, which the partial derivative is zero. 


$$
\left[\begin{array}{c}
\frac{\partial J}{\partial a_{1}} \\
\frac{\partial J}{\partial a_{2}} \\
\vdots \\
\frac{\partial J}{\partial a_{S}}
\end{array}\right]=\left[\begin{array}{c}
0 \\
0 \\
\vdots \\
0
\end{array}\right]
$$

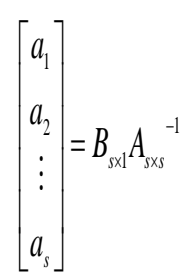

So the final fitting function is $P(x)=A^{\prime}-10 n^{\prime} \lg (x)$, where $A^{\prime}$ and $n^{\prime}$ are environmental parameters after fitting respectively.

2.3 Levy-PSO Localization. Flight Levy mechanism is a kind of Markov chain, which characterizes the step size that should meet the Levy condition. Where $n$ is the dimension of the solution, and the gamma function is $\Gamma(b)=\int_{0}^{\infty} \exp (-t)^{*} t^{b-1} d(t), \beta=3 / 2, \alpha$ is step size.

$$
\begin{aligned}
\left.\left.\sigma=\left(\Gamma(1+\beta)^{*} \sin (3.14 * \beta / 2) /\left(\Gamma((1+\beta) / 2) * \beta^{*} 2^{((} \beta-1\right) / 2\right)\right)\right)^{(1 / \beta)} \\
\\
u=\operatorname{rand}(n, 1) * \sigma \\
v=\operatorname{rand}(n, 1) \\
a=\alpha^{*}\left(u / a b s(v)^{1 / \beta}\right)
\end{aligned}
$$

On the basis of the particle swarm optimization algorithm, the random Levy flight mechanism is introduced in this paper to adjust the particle trajectory, which not only helps to get out of the local optimum, but also gets better convergence when the particles are in the global optimum. Its main function is that when the particles move in a small range near the local optimum, the jump of a large step can be given out. In the space of $D$ dimension, the formula for the velocity $V$ and the position $X$ of the particle at the time of $T$ is improved: $v^{d}(t+1)=v^{d}(t)+c 1^{*}\left(P i(t)-x^{d}(t)\right)+c 2^{*}\left(P g(t)-x^{d}(t)\right)$

$$
x^{d}(t+1)=x^{d}(t)+v^{d}(t+1)+\alpha^{*} \operatorname{sign}(\text { rand }-0.5) * \operatorname{levy}(\lambda)
$$

The specific steps of the PSO algorithm with the Levy flight mechanism are as follows:

(1) The initial solution and velocity value of each particle in the setting dimension are generated according to the size of the population;

(2) According to the fitness equation, the fitness value of each particle is calculated, and the optimal value of each particle is selected, and the optimal value of the particle is compared;

(3) Update the speed of each particle in accordance with the (9) formula given above, and limit the speed to a certain extent;

(4) Update the position information of each particle in accordance with the (10) given above, and place the position limit in the definition of the fitness function;

(5) When obtained fitness value is less than the required number of iterations and the number of iterations is not specific, and then return to step (2).

\section{Simulation and Test}

In order to describe the performance of the algorithm, we compare it with the standard PSO localization algorithm under different influences of communication distance and regional segmentation. In this simulation, the following testing conditions are assumed. The senor area is $60 \mathrm{~m} * 60 \mathrm{~m}$ and divided into several honeycomb regions. Senor nodes and anchors are distributed on the vertex of sub-region. The radius of honeycomb sub-region is set to $5 \mathrm{~m}$. The positioning interval of receiving node is $T_{s}=1$.

3.1 The Selection of Parameters. The effect of inertia weight is to keep the particle's motion inertia. Small w value can make particles to find the optimal solution in local space, while larger particles can search bigger space to find the global optimal solution. Figure 3 describes the inertia weight of the influence of average position error (Gaussian random variable is , the maximum number of iterations is ). It shows that the average error is significant when $\mathrm{w}$ is ranging from 0.1 to 0.5 . When w belongs to $0.5-0.7$, the average error is sharply decreasing. But when w value is over 
0.7 , the average error curve keeps smooth trend. Therefore, this paper uses the inertia weight $\mathrm{w}$ is 0.7 .

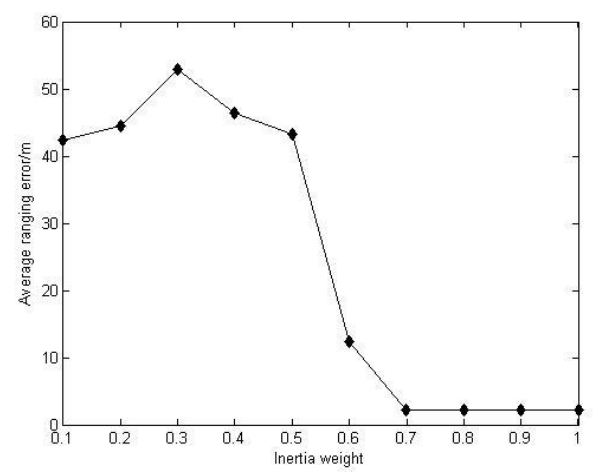

Figure 3. Average ranging error according inertia weight $\mathrm{w}$

The appropriate iteration number is helpful to shorten the algorithm running time and improve the efficiency of positioning. But the maximum iteration number often makes the algorithm cycle operation until the maximum number of iterations, which has the optimal value already. It reduces the real-time performance of the algorithm. When Gaussian random variable is $\lambda=N(0,9)$, inertia weight $w$ is 0.7 , measure different situation in the different maximum iteration number. Figure 4 shows that the average error is decreasing with the $M$ value are ranging from 50 to 200 . When $\mathrm{M}$ belongs to 200-500, the average error curve keeps smooth trend. Therefore, this paper uses the maximum iteration number $M$ is 300 .

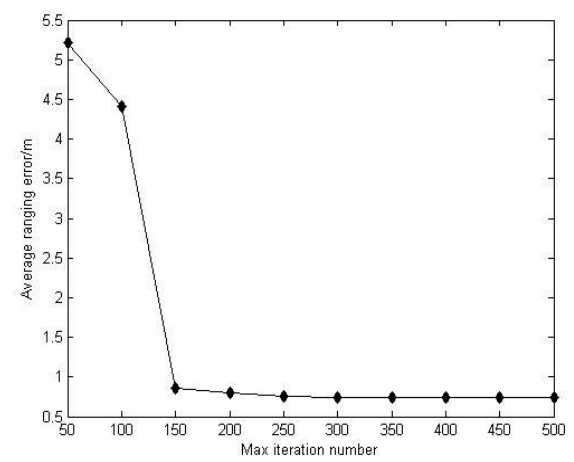

Figure 4. Average ranging error according max iteration number $M$

3.2 Communication Distance. The standard PSO localization algorithm based on RSSI without considering the influence of the communication distance. As is shown in Figure 5, there exists the relationship between communication distance and ranging error. The optimal communication distance is $5 \mathrm{~m}$. Thus we compare PSO algorithm and this article algorithm under the numbers of network connectivity and beacon nodes ratio.

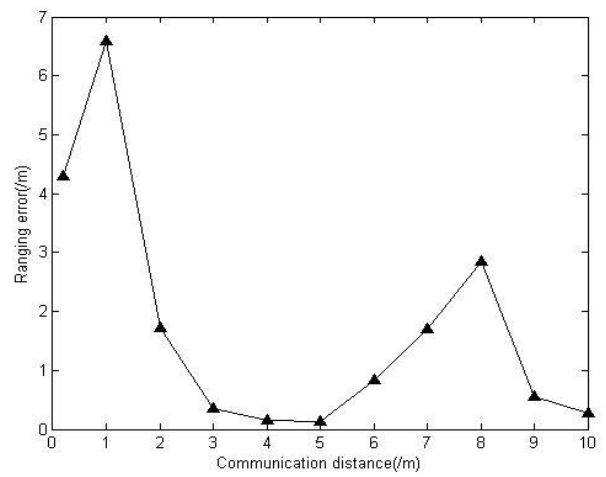

Figure 5. Relationship between communication distance and ranging error

(1) In order to obtain the index of network connectivity, we can adjust the communication beacon radius of nodes under the same number of nodes. Measure the RSSI values in every case of 10 times and average them. Because of the communication radius is changing randomly, the final result is the average positioning error ratio that is the mean divided by the average communication 
radius.

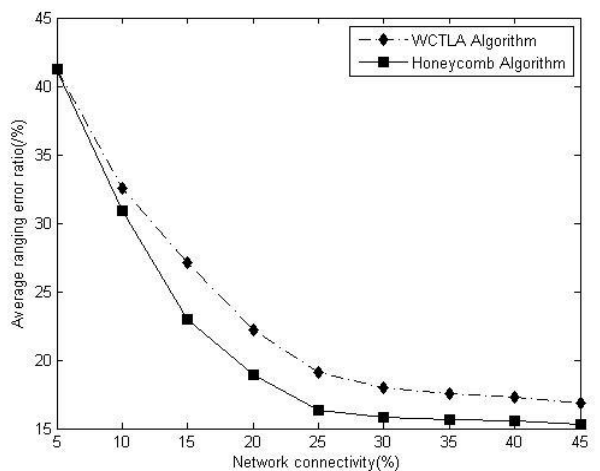

Figure 6. Average ranging error according network connectivity ratio

A performance comparison between the two algorithms shows the average ranging error in Figure 6. When the network connectivity is ranging from 5 to 25, the average ranging error of two algorithms has a sharply decreasing with the increase of network connectivity. On the contrast, the proposed algorithm will keep its location error bounded in a lower level when the network connectivity is over 25 . As a result, when the communication distance is over $5 \mathrm{~m}$, the curve of ranging error will tend to be smoothness. That is to show how too long communication distance cannot lower the ranging error.

(2) Furthermore the influence of the beacon nodes ratio on the average ranging error was investigated. Keep the same nodes number and nodes radius, the ratio of beacon nodes is accounted for $20 \%, 22.5 \%, 25 \% \ldots . . .50 \%$ to the total number. Test each case 10 times and the final result is the mean. Figure 7 presents the average ranging error drawn over the ratio of beacon nodes.

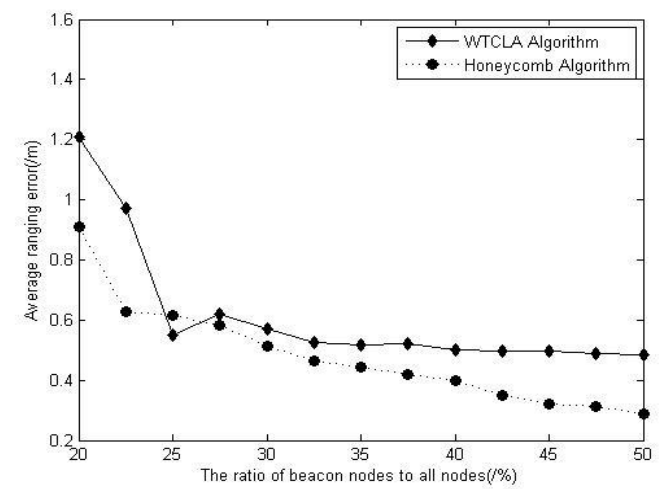

Figure 7. Average ranging error according beacon nodes ratio

Figure 7 presents the relationship between average ranging error and beacon nodes ratio. There is a sharp decrease from $30 \%$ to $50 \%$ when use the proposed algorithm. Due to the number of beacon nodes influence the hardware cost, the proposed algorithm is more adapted to the needs of indoor positioning.

3.3 Regional Segmentation. For triangle centroid localization algorithm, unknown nodes must communicate at least with three beacon nodes. Calculate the estimated distance based on three reference nodes to achieve positioning. However, the proposed algorithm reduces the positioning error caused by disturbances, which uses sub-regional environmental parameters respectively. Figure 7 is a comparison of three cases: (1) The triangle centroid algorithm, which develops the classic environmental parameters without weighted operation; (2) The WTCLA algorithm, developing the local environmental parameters and weighting the RSSI values; (3) This article algorithm, using the sub-regional environmental parameters and weighting the RSSI values. 


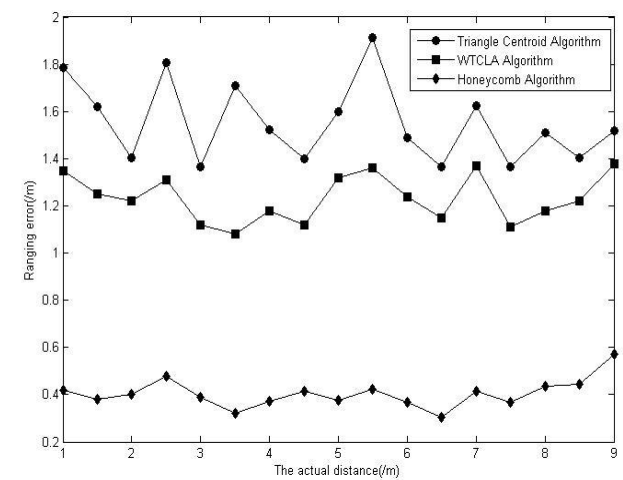

Figure 8. Ranging error according regional segmentation

From Figure 8, it can be observed that the regional segmentation has great influence on the localization performance of wireless senor nodes. When we developed the classical parameters in the whole testing area, the measured maximum is $1.38 \mathrm{~m}$ and the average error is $1.23 \mathrm{~m}$; however, the measured maximum is $0.57 \mathrm{~m}$ and the average error is $0.40 \mathrm{~m}$ when used the local environmental parameters. The positioning accuracy is increasing by $67.15 \%$ compared with the WCTLA algorithm. This shows that the classical environmental parameters cannot be applied to all sub-regions. Hence using the independent environmental parameters have a very significant effect on positioning error reducing.

\section{Conclusions}

This paper has proposed a honeycomb shape localization algorithm based on Levy-PSO. In this algorithm, the communication region of beacon nodes is divided into honeycomb sub-regions. Then sub-regional parameters use the least squares fitting and calculated optimal localization by PSO method based on Levy flight mechanism. Comparing to standard PSO algorithm, the proposed technique has higher accuracy and energy efficiency. The performance analysis of the proposed positioning scheme is also provided in the paper. In the future, we plan to combine other range-free algorithms and explore them into the honeycomb algorithm based on Levy-PSO.

\section{References}

[1] Akyildiz I., Su W. and Sankarasubramaniam Y., "A survey on sensor network", IEEE Communications Magazine, vol. 40, no. 8, (2002), pp. 102-144.

[2] Boukerche A., Oliverira H. A. and Nakamura E. F., "Location systems for wireless sensor network", IEEE Wireless Communications, vol. 14, no. 6, (2007), pp. 6-12.

[3] Niclescu, Americ N. L., "Communication paradigms for sensor networks", IEEE Communications Magazine, vol. 43, no. 3, (2005), pp. 116-122.

[4] S. Long, W. F. Bao, D. W. Jun, "Rang-free self-localization mechanism and algorithm for wireless sensor networks", Computed Engineering and Applications, vol. 23, (2004), pp. 127-130.

[5] L.R. Ren, J. Xiao, "The research of self-localization technology based on TOA in wireless sensor network", Information and Control, vol. 35, no. 2, (2006), pp. 280-283.

[6] S. Huajie, Z. Xiaoping, and W. Zhi, "Efficient closed-form algorithms for AOA based self-localization of sensor nodes using auxiliary variables", IEEE Transactions on Signal Processing, vol. 62, (2014), pp. 2580-2594.

[7] L. Z. Su, H. Z. Rong, "Particle swarm optimization with adaptive mutation", Acta Electronica Sinica, vol. 32, no. 3, (2004), pp. 416-420. 
[8] Z. S. Wang, W. Y. Long, G. Qiang, et al, "Particle Swarm optimization-based wireless sensor network nodes localization method", Journal of Shandong University Natural Science Edition, vol. 44, no. 9, (2009), pp. 52-55.

[9] Cura T, "Particle swarm optimization approach to portfolio optimization", Nonlinear Analysis: Real World Applications, vol. 10, (2009), pp. 2396-2406.

[10] Z. D. Xue, L. R. Quan, "Adaptive particle swarm optimization algorithm based on population velocity", Control and Decision, vol. 24, no. 8, (2009), pp. 1257-1260. 